\title{
Teleradiology: current perspectives
}

\author{
This article was published in the following Dove Press journal: \\ Reports in Medical Imaging \\ 8 January 2014 \\ Number of times this article has been viewed
}

\section{Elizabeth A Krupinski}

Department of Medical Imaging, University of Arizona, Tucson, AZ, USA
Abstract: Teleradiology is one of the oldest, most established, successful, and widely used clinical telemedicine specialties. There are a variety of factors that have led to this high degree of success, but there are also challenges and barriers that still exist. This review will address both of these aspects of teleradiology, within the context of the current literature on this topic, as well as from the perspective of a program that has been conducting teleradiology consultations for nearly 20 years. Some key considerations of what it takes to establish a successful teleradiology program will be discussed, with respect to technology, quality, and personnel. In particular, the role of mobile devices and smart phones will be reviewed, as these technologies are becoming much more pervasive throughout the health care enterprise. The important role of teleradiology in stroke assessment as an emerging application area will also be highlighted.

Keywords: standards, quality, telemedicine, technology, stroke assessment, telecommunications

\section{Introduction}

Radiology has undergone significant changes over the past 25 years or so as technology has changed both within the field and in areas such as advances and improvements in telecommunications. Within radiology, not only has the technology changed, but the expectations of the public in response to those changes have changed as well, in part, leading to the increased sub-specialization of radiologists along systems- and diseaserelated specialties. ${ }^{1,2}$ One consequence of this sub-specialization has been increased expansion and utilization of teleradiology, as clinicians and patients not only expect but often demand expert interpretation of images, not only in major urban, but in rural and medically underserved areas as well.

Teleradiology is well established, with on-call emergency reporting being used in over $70 \%$ of radiology practices in the US, and general teleradiology by "nighthawk services" around the world. ${ }^{2}$ The demand for imaging and subspecialty interpretation has also had the consequence of making radiology a 24/7 service that requires not just residents to be on-call after hours and on weekends, but the availability of expert attending-radiologists as well. Teleradiology, in part, makes this possible, and organizations such as the American College of Radiology (ACR $)^{1}$ and the European Society for Radiology $y^{2}$ encourage radiologists to make use of teleradiology services proactively that incorporate general and subspecialist radiologists through local area networks in areas where traditional onsite radiology services and expertise are lacking. In fact, the ACR recently updated their guidelines and standards for teleradiology practice calling for continued development of protocols and software to enable bidirectional communication between physicians, technologists, and imaging managers; better
Correspondence: Elizabeth A Krupinsk Department of Medical Imaging, University of Arizona, I609 N Warren BIdg 21 I, Tucson, AZ 85724, USA

Tel +I 5206264498

Fax +I 5206264376

Email krupinski@radiology.arizona.edu
Reports in Medical Imaging 2014:7 5-14 (c) (i) (5) 2014 Krupinski. This work is published by Dove Medical Press Limited, and licensed under Creative Commons Attribution - Non Commercial (unported, v3.0) License. The full terms of the License are available at http://creativecommons.org/licenses/by-nc/3.0/. Non-commercial uses of the work are permitted without any further permisson how to request permission may be found at: http://www.dovepress.com/permissions.php 
protocols for electronic medical record integration, peer review interfaces, and better dictation systems that eliminate manual interfaces. ${ }^{1}$

The ACR does however "believe the traditional practice model of having on-site, local radiology groups may better serve the overall interests of most communities." In part, this is due to the fact that although large commercial teleradiology practices do provide benefits in terms of overcoming geographic and coverage challenges, there is also the concern that they are commoditizing radiology by focusing only on report delivery and generating case volumes to improve their financial bottom line, ignoring the role of the radiologist as an integral expert member of the patient health care team. ${ }^{1}$ It should be noted, however, that there are many successful (and profitable) models for teleradiology outside the "commercial" sector both in academic and nonacademic settings, even though controversy still exists about its utility and role in the field of radiology in general..$^{3-11}$

What are some of the pros and cons? As noted, commercialization is one of the pros - to some extent the fear that radiologists will be nameless, faceless providers of interpretations without any contact with those receiving the reports - possibly losing their professional status with their non-radiology clinician peers. ${ }^{7}$ In a sense, radiology becomes no more than a service unit. To some extent this is true, although as Bradley ${ }^{6}$ notes, with the advent of picture archiving and communications systems (PACS), radiologists are seeing even less and less of their own in-hospital colleagues than ever before. With increased remote access to images via PACS and other patient data via the electronic health record, the changing distance between clinicians is more pervasive than just with teleradiology. Other pros include: not having to stay awake all night on-call, so when someone is called to provide a consult they are more alert and less likely to make mistakes; those working at night as "nighthawks" often receive more compensation and time off; those reading off-site emergency cases tend to hone their interpretation skills with these difficult cases and tend to emerge as expert readers; teleradiology can provide technical supervision for complex modalities such as computed tomography (CT) and magnetic resonance imaging (MRI); and it clearly provides access to subspecialist interpretation to those in geographically or otherwise remote or underserved areas. ${ }^{6,7}$

But what are some of the cons? For one, smaller radiology groups may be forced out of business as teleradiology companies move into the local market (although this may impact those with poorer customer service than others), potentially putting local radiologists out of work. ${ }^{7}$ It has also been noted that patients may not be aware that their images are not being read at the site they were acquired, potentially leading to increased concerns on their part (although there is little evidence to support this). ${ }^{7}$ Lower quality as a function of less control or knowledge of who is providing the interpretations and what their qualifications are has often been cited as a bad consequence of teleradiology. ${ }^{5,8,10}$ Encroachment by other specialties such as cardiology is also a potential consequence of teleradiology outsourcing. As Hunter et $\mathrm{al}^{3}$ note, however, many of these potential negative consequences can be minimized or even avoided by carefully considering the pros and cons of teleradiology and doing a thorough job of carefully evaluating a service provider before engaging their services.

The recent ACR teleradiology recommendations should be reviewed by anyone (both at the transmitting and receiving site) already involved in or just implementing teleradiology, as they cover such critical issues as the teleradiologist (licensure, malpractice, credentialing, Board certification, continuing medical education, peer review, and accreditation), the teleradiology work environment (ergonomics, monitor requirements, privacy, and security), interpretation services, medical record integration, communication, turnaround times, billing, payment, legal and regulatory considerations, and advice on contracts. ${ }^{1}$ For additional recommendations on technical considerations (eg, acquisition, transmission, storage, and viewing), the ACR-AAPM-SIIM Technical Standard for Electronic Practice of Medical Imaging was updated in 2012 and contains a considerable amount of relevant information (AAPM, American Association of Physicists in Medicine; SIIM, Society for Imaging Informatics in Medicine). ${ }^{12}$

\section{The global nature of teleradiology}

Teleradiology may have started primarily in the US, but it has spread dramatically across the world. ${ }^{13-16} \mathrm{~A}$ recent survey of European radiologists has revealed just how global teleradiology has become. ${ }^{13}$ The study involved 368 survey respondents and showed that $64.8 \%$ use teleradiology and $36.1 \%$ plan to do so soon. Although most teleradiology involves distribution of images in the same institution (70.9\%) (although it is debatable whether distribution within an institution is true teleradiology), $44.4 \%$ involves reading images from home overnight and on weekends. Image outsourcing is done by $35.5 \%$ to obtain expert opinions $(20.2 \%)$ or deal with capacity problems $(19.7 \%)$. Services are provided by $31.6 \%$ on a noncommercial basis and by $26 \%$ commercially, 
with most cases involving primary reads $(76.6 \%)$ and subspecialty interpretations $(50.5 \%)$. Some of the advantages noted by the respondents include the ability to discuss cases collaboratively (46.1\%), the ability to distribute workload (37.8\%), asking for second opinion and/or subspecialty advice $(81.9 \%)$, and increased availability of radiologists for on-call services $(71.3 \%)$. Some the disadvantages include insufficient integration of patient history and/or previous studies $(68.9 \%)$, limited communication with clinicians (67.7\%), possible loss of control of radiology businesses $(60.2 \%)$, possibly diminished quality of radiology services (54.4\%), instability in the job market and income (48.8\%), and a possible threat to good patient care (48.4\%). Suggestions to improve teleradiology quality include development of an obligatory quality assurance system (78.3\%), teleradiology training (70\%), and the use of double-reading $(56.9 \%) .{ }^{13}$

Teleradiology is also used widely by the military, not only in the US but around the world, in war and during peace. ${ }^{17-19}$ One reason why it has been so successful in the military is simply the environment and critical circumstances during military engagements. There are often not enough radiologists in the battle theater (where only limited imaging devices are feasible to use) or in the mobile medical units, so images must be acquired and transmitted from the theater of military operation to higher-level health care facilities (often overseas) for teleconsultation and telemanagement. Continuity of care is also critical once wounded warriors return home, as is general care for service members and their families during peacetime.

Currently, the US Army Medical Department Telehealth Network covers 50 countries and territories across 19 time zones. It covers 22 different services (with behavioral health accounting for $55 \%$, followed by cardiology, teledermatology, infectious disease, neurosurgery, pain management, and orthopedic surgery) in addition to teleradiology. ${ }^{19}$ Teleradiology is critical on the battlefield, where the deployable teleradiology systems consists of the Theater Image Repository. Images from the various theaters of operation are sent to Landstuhl US Army Base, Landstuhl, Germany which then sends them to US military treatment facilities. Trauma cases are readily handled by bundling images from the operating room for case conferences that discuss trauma care management for battlefield wounds. The teleradiology service is also used to provide maintenance of the CT scanners used in the theater, ensuring that they are functional when needed. Continuity of care is facilitated by the fact that the entire military medical system uses digital radiology and is connected by a uniform electronic health record (at least within each branch) that will eventually be integrated into a uniform system throughout the Department of Defense and the Veteran's Administration.

Teleradiology is also a key component in other medical services such as the growing application of teledentistry, ${ }^{20}$ and there are more studies emerging demonstrating its utility. For example, $\mathrm{Choi}^{21}$ recently investigated the clinical usefulness of teleradiology in general dental practice. In the study, 275 cases were submitted for inquiry to the case presentation board of the Korean Academy of Oral and Maxillofacial Radiology. The diagnoses were analyzed with respect to disease classification, correlation with the patient's chief complaint, whether additional examinations or treatments were needed, the image modalities, and the number of dentists making inquiries. They found that differential diagnoses of normal anatomic structures are the most frequently submitted cases (15.6\%). Overall, 164 cases require no additional treatments or exams. The most frequently submitted images are panoramic radiographs accompanying 248 of the inquiries. It was concluded that the teleradiology system could be helpful in the differential diagnosis of common dental lesions and would reduce unnecessary costs.

\section{The impact of teleradiology on radiologists and teleradiology practices}

Does the availability of teleradiology services impact referral rates and the types of cases referred? The answer is likely yes. For example, Tublin et $\mathrm{al}^{22}$ conducted a study to examine the impact of overnight subspecialty radiology coverage on CT utilization rates in academic and community emergency departments (EDs) for neuroradiology and abdominal imaging. The traditional overnight model in most academic centers is to have residents (usually senior) provide preliminary interpretations of CT studies, which are then over-read by attending radiologists the next day or in some cases by the on-call attending. In recent years, some academic centers have used teleradiology services to over-read the residents or to provide the primary interpretations. In the model adopted, rotating shifts of neuroradiologists and abdominal imagers provide contemporaneous final reports for ED CT studies from 5:00 pm to 7:00 am. The study compared total CT volume, ED visits, and CT volume/ED visits before and after implementation of overnight coverage. It was found that academic ED visits increased by $7 \%$ and community ED visits decreased by $3 \%$ during the study period. Total academic ED CT volume increased by $8 \%$, and community ED CT volume increased by $9 \%$. Daytime community ED CT volume remained constant, but on-call volume increased by $16 \%$. 
Academic ED CT volume/ED visits remained constant, but community increased. Basically, the overnight subspecialty ED coverage resulted in a disproportionate increase in $\mathrm{CT}$ utilization in the community EDs, perhaps because they lack in-house clinical subspecialists and need subspecialist radiology interpretations for patient management.

Another recent study examined whether teleradiology interpretation benefits from familiarity with referring physicians (ie, the teleradiology customers). ${ }^{23}$ The study tested the hypothesis that customer-specific experience would have a greater effect on individual performance than all other types of experience. The idea is that varied experience (ie, distribution of experience across multiple customers) may increase the rate of learning by an individual, for example, by identifying best practices from one customer that can be transferred to another, or by recognizing higher order principles that affect multiple customers. The study used data from Flatworld Solutions (Princeton, NJ, USA), a large US-based provider of teleradiology services using radiologists who are board certified and licensed to practice in the US (about $85 \%$ in the US and $15 \%$ overseas working from home or reading centers owned by Outsource Co). The dataset included 2,766,209 cases read by 97 radiologists for 1,431 customers over a 30-month period.

A key measure was length of time to read a case. They divided radiologist's prior experience into two categories: individual customer experience or the cumulative number of prior cases read by a radiologist for the customer of the current case; and cumulative number of prior cases read by a radiologist for all customers other than the customer of the current case. Domains were analyzed as well and were defined based on the body part imaged and categorized in accordance with subspecialties recognized by the ACR and the Radiological Society of North America (abdomen, body, brain, breast, cardiovascular, chest, gastrointestinal/genitourinary, head and neck, musculoskeletal, obstetrical, and spine).

The results support the benefits of customer-specific experience accumulated by individual radiologists, and variety in an individual's customer experience may increase the rate of individual learning from customer-specific experience for a focal task. Additionally, the level of experience with a customer for the entire outsourcing firm also produces learning, and experience across a wider variety of customers might assist the rate at which individuals learn from their own customer-specific experience. It is interesting to note that along these same lines, the European Society for Radiology recommends that all radiologists involved in teleradiology networks keep close contact with referring physicians through both personal interaction and video conferencing. ${ }^{2}$

\section{Security and privacy considerations}

Patient privacy and data security are key issues in any radiology service, ${ }^{24}$ so teleradiology is no exception. Although security and privacy have been incorporated into teleradiology practice nearly since its inception, there continues to be considerable research in this area looking for improvement. ${ }^{25,26}$ All teleradiology practices in the United Sates should be aware of the provisions in the 2009 Clinical Health Act (HITECH), Medicare and Medicaid initiatives for the adoption of and meaningful use of health information technology, what is entailed in electronic health records and technology by eligible providers, eligible hospitals, and/or critical access hospitals, as well as the 1996 Health Insurance Protection and Accountability Act regulations and updates. ${ }^{14}$ The ACR-AAPM-SIIM Electronic Practice Guidelines also provide some useful recommendations regarding security and privacy specifically with respect to medical imaging. ${ }^{24}$

There are a variety of ways to address privacy and security when dealing with patient data, and the requirements are for the most part defined by strict ethics and legislative rules. The basic international standard developed for security management of health information is the ISO27799 (Security Management in Health Using ISO/IEC/17799), which provides guidance on how to protect information via implementation of ISO17799/ISO27002. ${ }^{27}$ Digital Imaging and Communications in Medicine (DICOM) also addresses security and privacy issues, and there is continued research into improving the standard and optimizing its performance. ${ }^{28}$

For teleradiology, digital watermarking is becoming one of the more popular methods to protect images. Digital watermarking is a method of adding information to an image as a watermark (much like the watermark on a piece of paper or fifty dollar bill). The idea is that the watermark is inserted into the image before transmission so that it can be detected afterwards, and if the image has been tampered with in any way, the watermark will reveal it. It is essentially an authentication method. The watermark itself is typically not visible to the human eye and is not placed where it will interfere with image content (so as not to impact diagnoses if it is visible). Generally, digital watermarking has three major components, the water-mark generator, the embedder, and the detector. Methods differ, but they are being investigated intensely as more and more teleradiology images are being transmitted. ${ }^{26}$ 


\section{Image and interpretation quality}

Most teleradiology practices in developed countries where digital acquisition devices are more the norm than the exception (even in rural areas), tend to require that teleradiology images be only from digital acquisition devices. The main reason for this is that digitized images generally are of poorer quality which leads to reduced diagnostic confidence. ${ }^{3,29}$ When digitizers are used, there is evidence that for the most part digitizers are fairly equivalent in terms of acceptable contrast and low geometric distortion (even the less expensive non-radiology specific ones), thus there are circumstances where these solutions are acceptable..$^{30}$ Even with digitally acquired images, however, there is considerable variability in the quality of images between sites, both as a function of the basic imaging procedures and the equipment used, so users need to be aware of these variations and account for them during the interpretation process. ${ }^{31}$

Telemammography is even feasible, especially with the predominance of full-field digital mammography systems at least in developed countries. ${ }^{32,33}$ The Arizona Telemedicine Program is one example of a telemedicine program, which has even explored the utility of bundling telemammography, telepathology, and teleoncology services to create a new health care delivery pathway called the ultra-rapid breast care process. The aim is to reduce the time it takes for women to obtain diagnostic and therapeutic breast-care services from several weeks to a single day by utilizing telecommunications technology and digital mammography and pathology interpretations..$^{33}$ In the traditional care model, mammography (digital) occurs on day 1, with a report typically being available 2-7 days later. If a biopsy is needed, it can occur as early as day 8 (assuming the report was delivered on day 7), with a lab report available by about day 16 (and a second opinion if needed by day 24). On day 30 , there is an appointment with the surgeon to go over the surgery options, and on day 35 the surgery is scheduled. With the ultra-rapid model using telemammography and telepathology, all of the steps through the biopsy second opinion can occur within the same day, and the patient can even be connected in real-time to a teleoncologist to discuss the results and options. The entire process needs to be highly orchestrated and scheduled, but it is feasible, and patients are receptive. ${ }^{33}$

One question that always arises is whether the quality of teleradiology is equivalent to onsite services. As already noted, the ACR has some reservations, as do others in the field of radiology. ${ }^{1}$ There are, however, studies that do demonstrate that teleradiology can be effective, and quality can be high even using senior residents. ${ }^{1-11} \mathrm{~A}$ recent study by
Hohmann et $\mathrm{al}^{34}$ assessed the quality of their radiology outsourcing of after-hours CT. The study included a retrospective review of 1,028 in-house patients (at the Central London University Hospital) referred for imaging from 7:00 pm to 9:00 $\mathrm{pm}$. The cases were read by teleradiologists elsewhere in the United Kingdom or in Australia. The teleradiology reports were subsequently reviewed in the morning by in-house faculty radiologists using a five-point scale, where 5 was "no disagreement" and 1 was "definite omission or misinterpretation with unequivocal potential for serious morbidity or threat to life." The cases covered a wide range of types (eg, head, chest, and pelvis) and findings (eg, pulmonary embolism, bowel ischemia, and aortic dissection). In 79\% of the cases, there were no disagreements between onsite and teleradiology interpretations; in 16\% there was "disagreement over style and/or presentation of the report including failure to describe clinically insignificant features (rating $=4$ )"; in $4 \%$ there was "clinical significance of disagreement is debatable or likelihood of harm is low" (rating =3); and in 1.3\% there was "definite omission or interpretation of finding with strong likelihood of moderate morbidity but not threat to life" (rating =2). The category 2 cases were followed up, and it was found that of these, $8(0.8 \%)$ were correctly diagnosed by the in-house radiologist, $2(0.2 \%)$ by the teleradiologist, and $3(0.3 \%)$ were unresolved. Thus there was only a $0.8 \%$ proven serious misinterpretation rate. Other studies have found disagreement rates anywhere from $1.6 \%$ to $24.6 \%$ depending on the type of practice, modality, types of cases, and resident versus attending. ${ }^{35-41}$

\section{Mobile devices and smartphones}

The ACR-AAPM-SIIM Technical Standard for Electronic Practice of Medical Imaging 5 provides recommendations and minimum requirements with respect to traditional reading environment displays. However, it cannot be ignored that mobile devices such as tablet and smartphones have been the subject of research for a number of years and are now being used in a wide variety of medical applications, including imaging. The US Food and Drug Administration (FDA) has approved at least two viewers (mobile medical apps) for radiology (Resolution MD by Calgary Scientific, Inc Calgary, AB, Canada; and Mobile MIM by MIM Software, Cleveland, OH, USA), although the approvals do come with specific language regarding the types of images that can be viewed and under what circumstances (including ambient light considerations). ${ }^{42}$ Specifically, the guidance notes that "a mobile app that displays radiological images for diagnosis transforms the mobile platform into a class II Picture 
Archiving and Communications System (PACS) under 21 CFR 892.2050." ${ }^{\prime 2}$ Both of these products are considered Class II devices by the FDA. Class I devices are those with low to moderate risk and require general controls; while Class II devices are considered to have moderate to high risk and come with general controls and special controls.

For these mobile applications (Mobile MIM specifically), the special controls include the fact that it is not meant to be used in place of full workstations. The software also comes with a contrast test that determines if lighting conditions are sufficient to read images.

Exactly what "no access to a workstation" means is up to the user, as it is rather vague, but in general, most radiologists are aware of their limitations as well as those of display devices and hopefully will be using these devices only under suitable environmental conditions.

The real question for the radiologist (and the patient) is whether the diagnoses made using a mobile app and device is acceptable in terms of quality and under what circumstances can a primary read be given versus a second opinion or overread be given. The question is not an easy one, but studies on how to create mobile-based networks for teleradiology $25,43,44$ as well as those on diagnostic accuracy are becoming much more common in the literature, and these devices are being used for such applications as diagnostic reading, decision support, and access to medical books, interactive encyclopedias, and journal clubs. ${ }^{43}$

One of the first studies to evaluate iPhones (Apple Inc, Cupertino, CA, USA) for teleradiology was by Modi et al ${ }^{44}$ for the diagnosis of acute cervico-dorsal spine trauma. Using their own viewing software, they had two radiologists view a series of $75 \mathrm{CT}$ cases of patients suspected of having cervico-dorsal spine fractures, once on the iPhone and once on a traditional workstation. Sensitivity and accuracy for detecting vertebral body fractures was $80 \%$ and $97 \%$, and for posterior element fractures was $75 \%$ and $98 \%$ for one reader and $50 \%$ and $97 \%$ for the other one. There were no significant differences between the iPhone and workstation (although there were only a limited number of fractures in the study).

The same group was also one of the first to evaluate the Apple iOS (Apple Inc) for diagnosing acute stroke versus a workstation. They used 120 non-contrast $\mathrm{CT}$ brain scans and $70 \mathrm{CT}$ angiography scans, with one radiologist using an iOS device and the other using a workstation. ${ }^{45}$ Performance in terms of detecting acute parenchymal change, dense vessel signs, and vessel occlusion were all quite high, with high inter-reader agreement. Although both readers did not view the same images on both displays, their results were still favorable for mobile device reviewing of stroke images.

Another early study on this topic assessed the interpretation of orthopedic (fracture) and CT (intracranial hemorrhage) images using a personal digital assistant (PDA) and an iPod Touch (Apple Inc) device compared with an offthe-shelf secondary-class display monitor. ${ }^{46}$ Surprisingly, the PDA readings for the $\mathrm{CT}$ cases were significantly higher than with the monitor, and although some of the other comparisons trended to significance, they were all essentially equivalent for PDA and iPod versus the monitor. The study concluded that although there clearly needed to be more research, the results were promising for emergency use of mobile devices at least for these two types of cases and findings.

A more recent study assessed the more complicated scenario of interpreting $\mathrm{CT}$ cases for pulmonary embolism using an iPad (Apple Inc) versus a traditional PACS workstation. ${ }^{47}$ Although only two readers were used, it showed that both of them interpreted $98 \%$ of the cases correctly $(98 \%$ versus $100 \%$ sensitivity; $98 \%$ versus $96 \%$ specificity; $98 \%$ versus $98 \%$ accuracy). One factor supporting the utility of iPads for reading CT and MRI images is that iPad resolution is greater than what is actually required for these types of images. Further confirming their viability is a recent study by Bhatia et a ${ }^{48}$ comparing iPad $(1024 \times 768$ resolution $)$ versus standard PACS viewing $(1200 \times 1600$ resolution liquid-crystal display [LCD]) for acute central nervous system events (emergencyroom generated CT angiography perfusion imaging, brain MRI, and spine MRI), but using more readers and images. Overall, they found excellent intra-observer and inter-observer agreement for all three types of cases and for both radiologists and neurologists for assessing acute central nervous system injuries and other non-acute pathologies using the iPad. Other recent studies are also yielding results that indicate the utility of tablets, smartphones, and other mobile devices for interpreting certain types of radiographic images. ${ }^{49-51}$

From a practical point of view, one needs to consider the physical device itself. Current generations of cell phones and tablet devices have very high resolution ("retinal") displays that are actually nearly medical-grade. They also have quite a lot of memory, desktop-quality computational power (64-bit processors), and high bandwidth (4G LTE [long-term evolution]) with some service providers. When you think about it, the early days of digital radiology did not have displays and computers that were this good! However, although there is a trend these days towards smartphones with larger screens (some are almost the size of a tablet device), the majority of screens are still quite small and thus, even with 
high resolution, may not be especially well suited to reading radiographic images. Chest, bone, mammography, and other larger dimension images certainly require zooming and panning to view all image data at a resolution/size amenable to detecting findings, but this is clearly not the best solution, even for those quite facile with touch screens and manipulating images on these small devices. In the long-run it seems likely that given the controversies that abound with teleradiology and mobile devices, there will need to be guidance from the FDA, and Class I clearance may well be needed. ${ }^{52}$

\section{Teleradiology and telestroke assessment}

In 2009, the American Heart Association/American Stroke Association reviewed the evidence for the use of telemedicine within systems of care for stroke, and issued a scientific statement with regard to: 1) using FDA-approved teleradiology systems for review of brain CT in patients with suspected acute stroke, 2) brain CT scan reviews by stroke specialists or radiologists using FDA-approved teleradiology systems for identifying exclusions for thrombolytic therapy, and 3) FDAapproved teleradiology systems to support rapid imaging interpretation in time for thrombolysis decision-making. ${ }^{53}$ Due in part to this guidance document, telestroke services that incorporate teleradiology have rapidly expanded in the past few years, and the amount of research in this area further validating the utility and effectiveness of telestroke services has increased as well.

One important question is who should be interpreting the CT scans, as these are rather complex imaging studies, often with subtle findings that require some degree of expertise to detect and interpret accurately. A recent study by Demaerschalk et $\mathrm{al}^{54}$ was conducted to determine the degree of agreement between central reading committee neuroradiologists, spoke hospital radiologists, and hub hospital vascular neurologists (aka, telestrokologists) for the interpretation of baseline brain CT scans of patients with acute stroke presenting to telestroke spoke hospitals. The study was prospective, using an urban hub and two rural spoke sites, and was randomized, blinded, and controlled. The ultimate goal was to validate an audiovisual telemedicine and teleradiology system designed for remote evaluation of adult patients with acute stroke versus phone consultation to assess eligibility for intravenous thrombolysis treatment. For the telemedicine arm, CT scans were interpreted by the hub telestrokologist and on the phone by the spoke radiologist (total $n=54$ ). For the gold standard, the scans were interpreted independently and blindly by two hub neuroradiologists. The outcome was determination of a
CT-based contraindication to thrombolytic treatment. Overall, agreement was very high (kappa $=0.91$ ) and did not differ between telestrokologist and neuroradiologist (kappa $=0.92$ ) or spoke radiologist and neuroradiologist (kappa $=0.89$ ).

As discussed previously, the use of mobile devices is expanding in teleradiology, so it is logical to ask whether they can be used in telestroke interpretation as well. To address this, Demaerschalk et $\mathrm{al}^{55}$ evaluated the use of ResolutionMD ${ }^{\mathrm{TM}}$ (Calgary Scientific, Calgary, AB, Canada) - a mobile app for smartphones that gives vascular neurologists access to radiological images of stroke patients. The study assessed app use in a real telestroke network. The goal was to determine the degree of agreement between hub vascular neurologists using the ResolutionMD app, spoke radiologists using a PACS, and independent evaluators of brain CT scans. The study included 53 stroke patients. Agreement (kappa) between hub vascular neurologists using ResolutionMD and 1) the spoke radiologist, and 2) independent interpreters on a variety of clinically important features was overall quite high, including identification of intracranial hemorrhage (spoke radiologist 0.92-1.0; independent interpreters 0.93-1.0), neoplasm (spoke radiologist 0.92-1.0; independent interpreters $0.93-1.0$ ), any radiological contraindication to thrombolysis (spoke radiologist 0.92-1.0; independent interpreters $0.65-1.0$ ), early ischemic changes (spoke radiologist 0.28-0.96; independent interpreters $0.30-0.86$ ), and hyper-dense artery sign (spoke radiologist 0.01-0.80; independent interpreters 0.06-0.81).

A similar study by Park et al evaluated the interpretation of brain CT with subtle hemorrhage using an iPad 2 versus a clinical grade LCD monitor. ${ }^{56}$ They used 100 brain CT exams acquired for head trauma or headache, with 50 having subtle radiological signs of intracranial hemorrhage and 50 with no significant abnormality. Five emergency medicine physicians reviewed them. There was high sensitivity and specificity using both devices and ROC (receiver operating characteristic) areas under the curve were similar (0.935 and 0.900).

\section{Selecting a teleradiology service - important considerations}

When deciding whether or not to go with a teleradiology service or deciding which service to use once you have made the initial decision to use teleradiology, there are some important considerations to keep in mind. ${ }^{3}$ The first aspect concerns what exactly you want from a teleradiology service. This covers a variety of topics, including: final versus preliminary reads (cost and turnaround times would differ); whether or not resident reads would be acceptable, and if yes, would they require final reading by an attending; 
the types of cases to be covered; when you need coverage (eg, 24/7, nights only, weekends only, or only when local radiologists are on vacation); what types of critical (ie, lifethreatening findings on routine studies) findings require an emergency written report and personal notification; expected turnaround times; and whether reporting requirements differ for emergency versus routine cases.

Once a provider has been selected, the teleradiologists must be licensed and credentialed per federal, state, and local regulations. This is important, not only for legal reasons, but also for billing considerations as, for example, Medicare and Medicaid will only reimburse if the interpreting radiologist is on US soil. Ensuring that they are properly covered with adequate malpractice insurance is also important. In this vein, it is also useful to insist that the provider has a peer review process in place and that the results of that peer review be made available upon request. As already noted, there are specific guidelines in place for electronic practice of radiology and teleradiology $y^{1,12}$ but these should be reviewed with the provider to ensure for example that they adhere to the DICOM standard, ${ }^{57}$ the Health Level Seven standard, ${ }^{58}$ and the 1996 Health Insurance Protection and Accountability Act. ${ }^{59}$

On both the provider and referring sides, there should be specific contact people covering such aspects as contracts and billing, information technology, and clinical services, with responsibilities, contact information, and procedures clearly identified. It is also very useful for the teleradiologist to have the contact information for the radiology technologist(s) at the referring site, since as noted previously, poor quality images are often a problem with teleradiology interpretation. It is also very useful to have periodic onsite visits from the teleradiologist, not only for the personal contact, but also so that they can have a clear picture of the services, technology, staff, and even patient populations at the referring site.

Reimbursement is always a key consideration, and there are a variety of models being used. A very common model is for the teleradiologist (or teleradiology service) to be paid a set amount per case or per time period, with the amount being set as a function of modality, nature of the study, and urgent versus routine. In this case, it is useful for both sides to keep track of the cases and reconcile those numbers before payments are made. The referring contracting organization in the case is responsible for recovering its costs by billing insurers and/or patients. Another model is one in which the teleradiology provider bills directly. This is rather rare in the case of university-based teleradiology practices but is becoming a little more common as private practices venture into the teleradiology business.

\section{Conclusion}

It is clear that teleradiology has become embedded in the health care system and integrated into a wide variety of radiology practices and will likely continue to be the standard of care for many sites where onsite radiology is either limited or lacking completely. Although there are some concerns with respect to quality of service and commoditization of radiology, overall the benefits of teleradiology outweigh the potential negatives. As technology continues to evolve, we need to continue to conduct research that evaluates and validates these technologies before they are used routinely for patient care. As already described, teleradiology has extended beyond the radiology department into such applications as dentistry and stroke management, and it is likely that new applications will continue to be tested as needs arise and are identified. With the proper attention to optimizing technology, legal and regulatory guidelines, and common sense with respect to when and how teleradiology can and should be utilized, teleradiology will continue to grow, and its impact on patient care will continue to grow as well.

Where is teleradiology headed? It certainly is not going away, and it seems unlikely that the traditional radiology department will disappear either. There will always be the need for onsite radiologists (especially interventional), especially for training and education of residents and fellows. Additionally, although there have been changes in the frequency and type of contact between radiologists and other clinicians, there is still contact, especially with more complex cases. Sometimes the report simply requires that additional in-person explanation, where the radiologist can directly point to findings in the image and explain what they are seeing to the non-radiologist.

From the patients' perspective, having the radiologist available onsite may also be a reason we continue to see traditional radiology departments. Mammographers have always had close ties with their patients and traditionally are the clinicians that discuss findings with their patients rather than having the primary care physician or referring clinician talk with them. However, there is some evidence that in some circumstances (especially with positive findings), patients would prefer to hear the report from the radiologist expert rather than their primary care physician - and the majority would prefer to hear them in person rather than by phone or mail. ${ }^{60,61}$ With teleradiology, this would likely not be possible. In fact, recent guidelines from the ACR encourage improved communication with patients, including direct communication of results. ${ }^{62,63}$ 
Teleradiology is here to stay - although the exact form and conduct of it is likely to change as technologies change and the general practice of medicine changes. The current health care environment places a high premium on efficiency and increased access to quality care for all patients, and teleradiology represents one very important way that affordable health care will be available to all those that need it. The key is for users to be knowledgeable about the benefits and the costs (not just monetary) of teleradiology and to make informed decisions with the patient as well as the radiologist in mind. ${ }^{3}$

\section{Disclosure}

The author reports no conflicts of interest in this work.

\section{References}

1. Silva E, Breslau J, Barr RM, et al. ACR white paper on teleradiology practice: a report from the Task Force on Teleradiology Practice. J Am Coll Radiol. 2013;10:575-585.

2. European Society of Radiology. The future role of radiology in healthcare. Insights Imaging. 2010;1:2-11.

3. Hunter TB, Krupinski EA, Weinstein RS. Factors in the selection of a teleradiology provider in the United States. J Telemed Telecare. 2013;19:352-357.

4. Rosenberg C, Kroos K, Rosemberg B, Hosten N, Flessa S. Teleradiology from the provider's perspective - cost analysis for a mid-size university hospital. Eur Radiol. 2013;23:2197-2205.

5. Thrall JH. Teleradiology: two-edged sword or friend of radiology practice? J Am Coll Radiol. 2009;6:73-75.

6. Bradley WG Jr. Off-site teleradiology: the pros. Radiology. 2008;248: 337-341.

7. Boland GW. Teleradiology coming of age: winners and losers. AJR Am J Roentgenol. 2008;190:1161-1162.

8. Brant-Zawadzki M. The goose and the nighthawk: a bedtime fable for young radiologists (with apologies to the Brothers Grimm). J Am Coll Radiol. 2006;3:231-232.

9. Bradley WG Jr. Special focus: outsourcing after hours radiology another point of view: use of a nighthawk service in an academic radiology department. J Am Coll Radiol. 2007;4:675-677.

10. Levin DC, Rao VM. Outsourcing to teleradiology companies: bad for radiology, bad for radiologists. J Am Coll Radiol. 2011;8:104-108.

11. Kaye AH, Forman HP, Kapoor R, Sunshine JH. A survey of radiology practices' use of after-hours radiology services. J Am Coll Radiol. 2008;5:748-758.

12. Norweck JT, Seibert JA, Andriole KP, et al. ACR-AAPM-SIIM Technical Standard for Electronic Practice of Medical Imaging. J Digit Imaging. 2013;26:38-52.

13. Ranschaert E, Binkhuysen FB, Ziekenhuis JB. European teleradiology now and in the future: results of a survey. Int J CARS. 2012;7:S97-S102.

14. Marti-Bonmati L, Morales A, Bach LD. [Toward the appropriate use of teleradiology]. Radiologia. 2012;54:115-123. Spanish.

15. Kazley AS, McLeod AC, Wager KA. Telemedicine in an international context: definition, use, and future. Adv Health Care Manag. 2012;12: 143-169.

16. Venco $\mathrm{S}$. The emergence of a new medical model: the international division of labour and the formation of an international outsourcing chain in teleradiology. Work Org Labour Globaliz. 2012;6:45-57.

17. Cenname G, D’Ambrosio ID, Ajello C. Teleradiology: case series and experience acquired in the military field. Radiol Med. 2013;118: 688-699.
18. Beck JJW. Trauma imaging in and out of conflict: a review of the evidence. Radiography. 2012;18:292-295.

19. Poropatich R, Lai E, McVeigh F, Bashshur R. The US Army Telemedicine and $\mathrm{m}$-Health Program: making a difference at home and abroad. Telemed J E Health. 2013;19:380-386.

20. Marino R, Ghanim A. Teledentistry: a systematic review of the literature. J Telemed Telecare. 2013;19:179-183.

21. Choi JW. Clinical usefulness of teleradiology in general dental practice. Imaging Sci Dent. 2013;43:99-104.

22. Tublin M, Brown C, Pacella C, Tublin D, Yealy D. Overnight subspecialty radiology coverage: review of a practice model and analysis of its impact on CT utilization rates in academic and community emergency departments. AJR Am J Roentgenol. 2012;199:623-626.

23. Clark JR, Huckman RS, Staats BR. Learning from customers: individual and organizational effects in outsourced radiological services. Organization Sci. 2012;24:1539-1557.

24. Relyea-Chew A. Major regulatory changes and the impact on diagnostic imaging in the United Sates 2005 to 2012. Acad Radiol. 2013;20:1063-1068.

25. Satoh H, Niki N, Eguchi K, et al. Teleradiology network system on cloud using the web medical image conference system with a new information security solution. Proc SPIE Med Imaging. 2013;8674:86740X-1.

26. Nyeem H, Boles W, Boyd C. A review of medical image watermarking requirements for teleradiology. J Digit Imaging. 2013;26:326-343.

27. ISO [homepage on the Internet]. ISO 27799:2008 Health informatics information security management in health using ISO/IEC 27002. Geneva: International Organization for Standardization. Available from: http:// www.iso.org/iso/catalogue_detail?csnumber=41298. Accessed December 1, 2013.

28. Koutelakis GV, Anastassopoulos GK, Lymberopoulos DK. Application of a multiprotocol medical imaging communications and an extended DICOM WADO service in a teleradiology architecture. Int J Telemed Appl. 2012;2012:271758.

29. Krupinski EA, McNeill K, Ovitt TW, Alden S, Holcomb M. Patterns of use and satisfaction with a university-based teleradiology system. J Digit Imaging. 1999;12:166-167.

30. Salazar AJ, Camacho JC, Aguirre DA. Comparison between different cost devices for digital capture of X-ray films: an image characteristics detection approach. J Digit Imaging. 2012;25:91-100.

31. Abramson RG. Variability in radiology practice in the United States: a former teleradiologist's perspective. Radiology. 2012;263:318-322.

32. Fruehwald J, Jantsch M, Pinker K, et al. Teleradiology with uncompressed digital mammograms: clinical assessment. Eur J Radiol. 2013;82:412-416.

33. Weinstein RS, Lopez AM, Barker GP, Krupinski EA, Descour MR, Scott KM. The innovative bundling of teleradiology, telepathology, and teleoncology services. IBM Syst J. 2007;46:69-84.

34. Hohmann J, de Villiers P, Urigo C, Sarpi D, Newerla C, Brookes J. Quality assessment of out sourced after-hours computed tomography teleradiology reports in a Central London University Hospital. Eur J Radiol. 2012;81:e875-e879.

35. Agrawal A, Agrawal A, Pandit M, Kalyanpur A. Systematic survey of discrepancy rates in an international teleradiology service. Emerg Radiol. 2011;18:23-29.

36. Wong WS, Roubal I, Jackson DB, Paik WN, Wong VK. Outsourced teleradiology imaging services: an analysis of discordant interpretation in 124,870 cases. J Am Coll Radiol. 2005;2:478-484.

37. Platts-Mills TF, Hendey GW, Ferguson B. Teleradiology interpretations of emergency department computed tomography scans. J Emerg Med. 2010;38:188-195.

38. Stevens KJ, Griffiths KL, Rosenberg J, Mahadevan S, Zatz LM, Leung AN. Discordance rates between preliminary and final radiology reports on cross-sectional imaging studies at a level 1 trauma center. Acad Radiol. 2008;15:1217-1226.

39. Strub WM, Vagal AA, Tomsick T, Moulton JS. Overnight resident preliminary interpretations on CT examinations: should the process continue? Emerg Radiol. 2006;13:19-23. 
40. Filippi CG, Schneider B, Burbank HN, Alsofrom GF, Linnell G, Ratkovits B. Discrepancy rates of radiology resident interpretations of on-call neuroradiology MR imaging studies. Radiology. 2008;249: 972-979.

41. Briggs RH, Rowbotham E, Johnstone AL, Chalmers AG. Provisional reporting of polytrauma CT by oncall radiology registrars. Is it safe? Clin Radiol. 2010;65:616-622.

42. US Food and Drug Administration. Mobile Medical Applications: Guidance for Industry and Food and Drug Administration Staff. Silver Spring, MD: Food and Drug Administration; 2013. Available from: http://www.fda.gov/downloads/MedicalDevices/ DeviceRegulationandGuidance/GuidanceDocuments/UCM263366. pdf. Accessed October 15, 2013.

43. Szekely A, Talanow R, Bagyi P. Smartphones, tablets and mobile applications for radiology. Eur J Radiol. 2013;82:829-836.

44. Modi J, Sharma P, Earl A, Simpson M, Mitchell JR, Goyal M. iPhonebased teleradiology for the diagnosis of acute cervico-dorsal spine trauma. Can J Neurol Sci. 2010;37:849-854.

45. Mitchell JR, Sharma P, Modi J, et al. A Smartphone client-server teleradiology system for primary diagnosis of acute stroke. J Med Internet Res. 2011;13:e31.

46. Toomey RJ, Ryan JT, McEntee MF, et al. Diagnostic efficacy of handheld devices for emergency radiologic consultation. AJR Am J Roentgenol. 2010;194:469-474.

47. Johnson PT, Zimmerman SL, Heath D, et al. The iPad as a mobile device for CT display and interpretation: diagnostic accuracy for identification of pulmonary embolism. Emerg Radiol. 2012;19:323-327.

48. Bhatia A, Patel S, Pantol G, Wu YY, Plitnikas M, Hancock C. Intra and inter-observer reliability of mobile tablet PACS viewer system vs standard PACS viewing station-diagnosis of acute nervous system events. Open J Radiol. 2013;3:91-98.

49. Abboud S, Weiss F, Siegel E, Jeudy J. TB or not TB: interreader and intrareader variability in screening diagnosis on an iPad versus a traditional display. J Am Coll Radiol. 2013;10:42-44.

50. Rodrigues MA, Visvanathan A, Murchison JT, Brady RR. Radiology smartphone applications; current provision and caution. Insights Imaging. 2013;4:555-562.

51. Ege T, Kose O, Koca K, Demiralp B, Basbozkurt. Use of the iPhone for radiographic evaluation of the hallux valgus. Skeletal Radiol. 2013;42:269-273.

52. US Food and Drug Administration. FDA Medical Image Communications Device Guidance. http://www.accessdata.fda.gov/scripts/cdrh/cfdocs/ cfcfr/CFRSearch.cfm?fr=892.2020. Accessed December 1, 2013.
53. Schwamm LH, Holloway RG, Amarenco P, et al. American Heart Association Stroke Council; Interdisciplinary Council on Peripheral Vascular Disease. A review of the evidence for the use of telemedicine within stroke systems of care: a scientific statement from the American Heart Association/American Stroke Association. Stroke. 2009;40: 2616-2634

54. Demaerschalk BM, Bobrow BJ, Raman R, et al. CT interpretation in a telestroke network: agreement among a spoke radiologist, hub vascular neurologist, and hub neuroradiologist. Stroke. 2012;43: 3095-3097.

55. Demaerschalk BM, Vargas JE, Cannner DD, et al. Smartphone teleradiology application is successfully incorporated into a telestroke network environment. Stroke. 2012;43:3098-3101.

56. Park JB, Choi HJ, Lee JH, Kang BS. An assessment of the iPad 2 as a CT teleradiology tool using brain CT with subtle intracranial hemorrhage under conventional illumination. J Digit Imaging. 2013;26: 683-690.

57. NEMA [homepage on the Internet]. The DICOM standard. Rosslyn, VA: NEMA [updated 2009]. Available from: http://medical.nema.org/ standard.html. Accessed October 15, 2013.

58. Health Level Seven International [homepage on the Internet]. Available from: http://hl7.org. Accessed October 15, 2013.

59. HHS.gov [homepage on the Internet]. Health information privacy. Washington, DC: US Department of Health and Human Services. Available from: http://www.hhs.gov/ocr/privacy/hipaa/enforcement/ sag/index.html. Accessed October 16, 2013.

60. Kuhlman M, Meyer M, Krupinski EA. Direct reporting of results to patients: the future of radiology? Acad Radiol. 2012;19:646-650.

61. Basu PA, Ruiz-Wibbelsmann JA, Spielman SB, et al. Creating a patientcentered imaging service: determining what patients want. AJR Am J Roentgenol. 2011;196:605-607.

62. My Patient Connection [homepage one the Internet]. Reston, VA: American College of Radiology. Available from: http://www.mypatientconnection.com/Articles/NeimanLetter.aspx. Accessed December 1, 2013.

63. American College of Radiology. ACR practice guideline for communication of diagnostic imaging findings. http://www.guideline.gov/content. aspx?id=32541. Accessed December 1, 2013.
Reports in Medical Imaging

\section{Publish your work in this journal}

Reports in Medical Imaging is an international, peer-reviewed, open access journal publishing original research, reports, reviews and commentaries on all areas of medical imaging. The manuscript management system is completely online and includes a very quick and fair peer-review system, which is all easy to use.

\section{Dovepress}

Visit http://www.dovepress.com/testimonials.php to read real quotes from published authors. 